\title{
Usage of Enterprise Modeling Processes and Information Systems Design to Forecast Demand
}

\author{
Luciana Rocha dos Santos ${ }^{1}$, Simone Vasconcelos Silva ${ }^{2}$ and Renato de \\ Campos $^{3}$ \\ 1. Estácio de Sá University, Avenida 28 de Março, 423 - Centro - Campos \\ dos Goytacazes - Brazil, lurochas@yahoo.com.br, \\ www.estacio.br \\ 2. Federal Center for Technological Education of Campos, Rua Dr. \\ Siqueira, 273 - Parque Dom Bosco - Campos dos Goytacazes - Brazil, \\ simonevs@cefetcampos.br,www.cefetcampos.br \\ 3. São Paulo State University, Av. Eng. Edmundo Carrijo Coube, nº 14-01 \\ - Bauru - Brazil, rcampos@feb.unesp.br, www.feb.unesp.br
}

\begin{abstract}
Managing the great complexity of enterprise system, due to entities numbers, decision and process varieties involved to be controlled results in a very hard task because deals with the integration of its operations and its information systems. Moreover, the enterprises find themselves in a constant changing process, reacting in a dynamic and competitive environment where their business processes are constantly altered. The transformation of business processes into models allows to analyze and redefine them. Through computing tools usage it is possible to minimize the cost and risks of an enterprise integration design. This article claims for the necessity of modeling the processes in order to define more precisely the enterprise business requirements and the adequate usage of the modeling methodologies. Following these patterns, the paper concerns the process modeling relative to the domain of demand forecasting as a practical example. The domain of demand forecasting was built based on a theoretical review. The resulting models considered as reference model are transformed into information systems and have the aim to introduce a generic solution and be start point of better practical forecasting. The proposal is to promote the adequacy of the information system to the real needs of an enterprise in order to enable it to obtain and accompany better results, minimizing design errors, time, money and effort. The enterprise processes modeling are obtained with the usage of CIMOSA language and to the support information system it was used the UML language.
\end{abstract}




\section{Introduction}

Over the last years, deep changes have affected the competitive enterprise environment. Manage the production became a very harder task due to the necessity of integration of high qualified human resource, parallel to the competitive strategies which add value and improve productivity in the enterprise developing operations.

Furthermore, the global competition and the outsourcing have caused the fragmentation of the supplies chains, making the enterprises seek for collaboration with others through global conectiviness in order to find potential businesses and establish concrete business patterns [1,2]. To control all these business world variants is a high-risk and complex undertaking and it needs great capital investment, and to have its effect minimized it needs an adequate use of methodologies.

Open system architecture for computer-integrated manufacturing (CIMOSA) [3, $4]$ is one of the greatest initiatives in the research of enterprise integration. This architecture establishes a set of concepts and a model-based integration approach. The general, partial and particular principles enable the creation of reference models, or general solution which allows testing, adjusting and adaptations in agreement to the enterprise reality.

In this study to make the matter clearer and to exemplify the modelling process it will be detached only one area of the company: the demand forecasting. Generally, in small-to-medium enterprises, this subject area is very regardless due to the limited capacity of their investments, the little knowledge of their managers and also the fragility of the organization in the productive processes.

Based on a general model to the demand forecasting proposed by Santos [5], this article detaches the usage of enterprise models in the orientation design of an information system to aid the demand forecasting, using the reference models CIMOSA and UML for modelling information system. Its also describes briefly the functioning of an implemented prototype, as well as the difficulty of transporting the world enterprise pieces of information to an information system.

This article is structured as follows. Section 2 examines the aspects of the enterprise modelling. Section 3 discourses about the necessity of information in the enterprise decision-making. Section 4 introduces a general view of the demand forecasting. Section 5 deals with modelling systems. Section 6 describes the process models of the demand forecasting and the support information system. Section 7 presents some consideration about the new technologies and section 8 final considerations, as well as future works.

\section{Enterprise Modelling}

Designing the enterprise involves different enterprise models, each one detaching features and ignoring others. The enterprise modelling generally consists of several models such as: product, resource, activity, information, organization, economical 
and some decision structures, however, for their development some techniques and support tools are needed.

Through a model it is possible to plan, obtain procedures and documentation consistently show the enterprise reality, accomplish modifications in accordance with the desirable future situation, and incorporate the knowledge and technologies really necessary to the business with more security.

Enterprise modelling can be defined as the art of externalising knowledge, that is, it adds value to the enterprise which needs to be shared. And in order to detach the use of enterprise modelling follows a concise background along the run [6]:

- Mid-70's: SADT, ER model, DFD, semantic nets (IT appli. dev't)

-80's: CIM methods (ICAM-IDEF, GRAI, CAM-I)

- Late 80's: EU AMICE's CIMOSA / BPR (process orientation)

- Over the 90's:

- ERP deployment (DEM, ARIS, ...)

- Workflow management systems (WPDL)

- Object orientation (IEM, UML, ...)

- Ontologies (IDEF5, TOVE, Enterprise Ontology, PSL, i*)

Vernadat [6] also detaches some modelling tools which implement the above introduced methodologies, accordingly with the following classification:

- Commercial EM tools: ARIS Toolset, FirstSTEP, Bonapart, KBSI tools, PrimeObject, Enterprise Modeler, MO2GO, emaGIM, CimTool, ...

-Workflow Management tools: IBM Flow Mark, Oracle Workflow, Ultimus, WorkParty, Ensemble, InConcert, Action Workflow, OPEN/Workflow, Staffware, Lotus Notes, ...

CIMOSA is still one of the most comprehensive approaches to the development of enterprise models making possible the attainment of an integrated enterprise model which captures and structures the essential characteristics of the enterprise, besides supplying conditions for infrastructure that has supported the integration of the operations of the enterprise. Its objective is to cover essential enterprise aspects in one integrated model. These aspects, in brief, include business processes that compound the enterprise such as: function (business process), information (necessary to business process, as order), resources (necessary to the business process, as people and tools) and organization (the enterprise structure, as enterprises, departments, units and cells). Beyond reaching great flexibility with the instantiation principle, where generic building blocks can be detailed forming the partial model named reference architecture which can be particularized in accordance with the specific documentation of each enterprise [3, 7, and 8].

To deal with the current variants of the business world it is necessary to find convenient techniques, study, analyze, test and propose solutions. The enterprise 
model will enable an information system design with greater consistency, once all the reality of the enterprise will be able to be surveyed and the true business necessities known, organized and registered through the model.

\section{Enterprise Decision Making and the Need of Information}

Nowadays, to keep the integration of the several enterprise areas and to integrate its internal and external environments involve some connectiviness such as: internet, extranet, palmtops, and mobiles. Enterprises to become more competitive establish relationships among other companies creating new structures of business. This highlights the potential of the Internet to redesign enterprises in a panorama dominated by dynamic business relationships [2].

This scenario requires enterprises to be able to integrate and coordinate business processes, which go beyond their limits involving several autonomous entities. However, many organizations, especially, the small-to-medium enterprises, are reluctant to invest time and money in models to support decision making [5]. Extracting intelligent strategies and decision support tools need to be considered. The use of reference models can help to extend and support many decision strategies as production optimization, sells analysis, development of new products and services, investment analysis and the adoption of technologies [9].

The necessity of information and integration of the several enterprise areas normally depends on diverse factors such as: problem featuring, impact comprehension and identification. To capture these pieces of information reflecting the way they are found in the enterprise is a must to model them.

\section{Systems Modelling}

A model can be used to articulate with precision the necessities of the enterprise information and show a great deal of the processing logic which is implicit and not declared to the users. The difficulty in transporting the situation of a real world into a computing system is in leading with the complexity and the number of variable involved. To establish, give priority and connect these variants in way that it can represent faithfully the problem.

The vocabulary and the rules of the UML (Unified Modelling Language) $[10,11]$ indicate how to create and read well-formatted models, but they do not point out which models should be created nor when one should create them. The object is the main building block of all systems. A modelling language has the target turned back to the conceptual and physical representations of a system.

The CIMOSA generic constructs of the modelling language are provided in textual description and with templates, containing header, attributes and 
relationships. An overview of the modelling language constructs and their relationships is given in an UML Meta model graphical representation [12].

The models describes in this study are obtained from the enterprise models built with CIMOSA, which captures all the information necessary and after analysis filters the necessary information to compose the information system model UML.

\section{Demand Forecasting}

In the enterprise environment the demand forecasting is important, especially for the rationalization of the resources and for the correct planning of the client answering level. All planning activity requires forecasting about a future fact; if the forecasting becomes better, better will be the used hypothesis to the planning.

Enterprises, mainly the small-to-medium size, do not make the product demand forecasting as practice integrated from their habitual procedures. In some cases it is used only qualitative procedures and opinions. Great part of the enterprises due to intense daily and the lack of capacity possess a distorted understanding of the involved knowledge in the forecasting, executing activities that do not give potential results.

In accordance with Nikolopoulos and Assimakopoulos [13] the forecasting to enterprise represents one of the crucial factors with the aim to improve the performance of several industry operations and managements and thus help to ensure the efficient resource usage. It can create advantages for the enterprise with the addition of intelligent features as support and decision tools.

Santos [5] found the main problems to low the application of demand forecasting in small-to-medium enterprises: (i) based only in the opinion of analysts (not always enabled to this function); and (ii) difficulty to adjust qualitative questions, opinions, with quantitative questions obtained with the application of mathematical models.

The forecasting is an indispensable piece of information to the production planning, sales and finance of an enterprise, allowing the managers to foresee the future and plan adequately the actions to the development of capacity plans, cash flow, sales, production, storage, work force, purchases, and so on. An information system to forecast demand of a product will be able to provide an understanding and accompaniment of the forecasting, so guaranteeing a consistent base, but flexible through the usage of the resources and devices that adapt themselves to the different realities of the enterprise.

\section{Modelling Demand Forecasting and Information System}

To model the demand forecasting of products is a sufficiently difficult task since it is an area of the enterprise with diverse characteristics that depend on the size and 
segment, beyond other factors as: economic, quantitative data, opinions of specialists and integration with other areas of the enterprise.

Enterprise modelling consists of understand the essential features of a system and writing these down in a systematic manner [3]. This work presents a reference model [5], taken from a bibliographical review. The survey and the organization of this environment of generic forecast were obtained with application of the CIMOSA language. The expected one is formalisation of the involved knowledge in the demand forecasting of products promoting the reengineering of the processes and the construction of the information system.

After the capture of requirements and description of the business process in CIMOSA constructors, the object views are all analysed to derive the conceptual schema of the supporting information system using UML models. The UML is a powerful and analytical technique in easy-to-use or user-friendly languages which could be readily applied by business users. The obtained reference model elaborated follows a sequence of development and had two mains activities: Enterprise Model and Model Information System.

Enterprise Model - it uses CIMOSA language to define generic operational environment forecasting for the enterprise, or either, the domain forecast and its main components, processes, relationships, activities and information:

1) Requirements Definition Modelling (RDM) - in this level it captures the essential needs. The mains steps are to identify and to define:

Domain of the work - Domains (DMi);

- $\quad$ Elementary processes of each domain - Domain processes (DPi);

- Activities that compose each domain process - enterprise activities (EAi);

- To establish the input and output information for each activity of enterprise Object view (OVi).

2) Project Modelling Specification (PMS) - in this level of the modelling the RDM is expanded (DMi, DPi, EAi, OVi) the constructors are detailed.

Model Information System - extended the PMS and it uses UML language defining the conceptual schema:

1) From the enterprise model (PMS), it must be found and described actions that produce results of value for the system and who carries through each action. They are defined: Use Case Diagram and description use cases.

2) Based in the Enterprise Activities and Object View (PMS), and in the use cases description. The object class and relationships had been represented in the Class Diagram.

3) The detailed analysis of models PMS, Class Diagram and Use Case Model assists in the specification of the Sequence Diagram.

The environment of the demand forecast frequently involves two main areas of the company such as: the (i) PCP (Production Control Planning), for example, to plan the productive system (Plan production elaboration, to define goods and services to be offered to the market, to install equipment and to make use of work power) as well as to planning the use of the productive system (to organize production plans, storage, purchase plans, supply replacement, of the production 
sequencings); and the (ii) area of the marketing and sales department that has objectives such as: make decisions with relation to the promotion and discounting for amount, or launching of new product, or investment in advertisement, or penetration of new markets.

Fig. 1. Represents the forecast model concentrated in four great main processes: the Register of data, the Design of the forecast, the Demand forecasting and the Accompanying of the errors of the forecasting. The support information system to the demand forecasting implements the procedure considered in the Fig 1. The procedure begins from a request of the demand forecasting for one determined end (as to prepare a forecasting for one determined products family). This request initiates a forecasting design where some activities must be executed in order to prepare the forecasting.

The model is flexible allowing in accordance with management analysis, adjustments in the forecasting design, in the demand data, in the forecasting and the calculation of the errors, in order to get the demand forecasting closer to reality as possible.

In the process Register demand data, the data used in the demand forecasting must represent the demand for the product, which is not always compared to occurred sales.

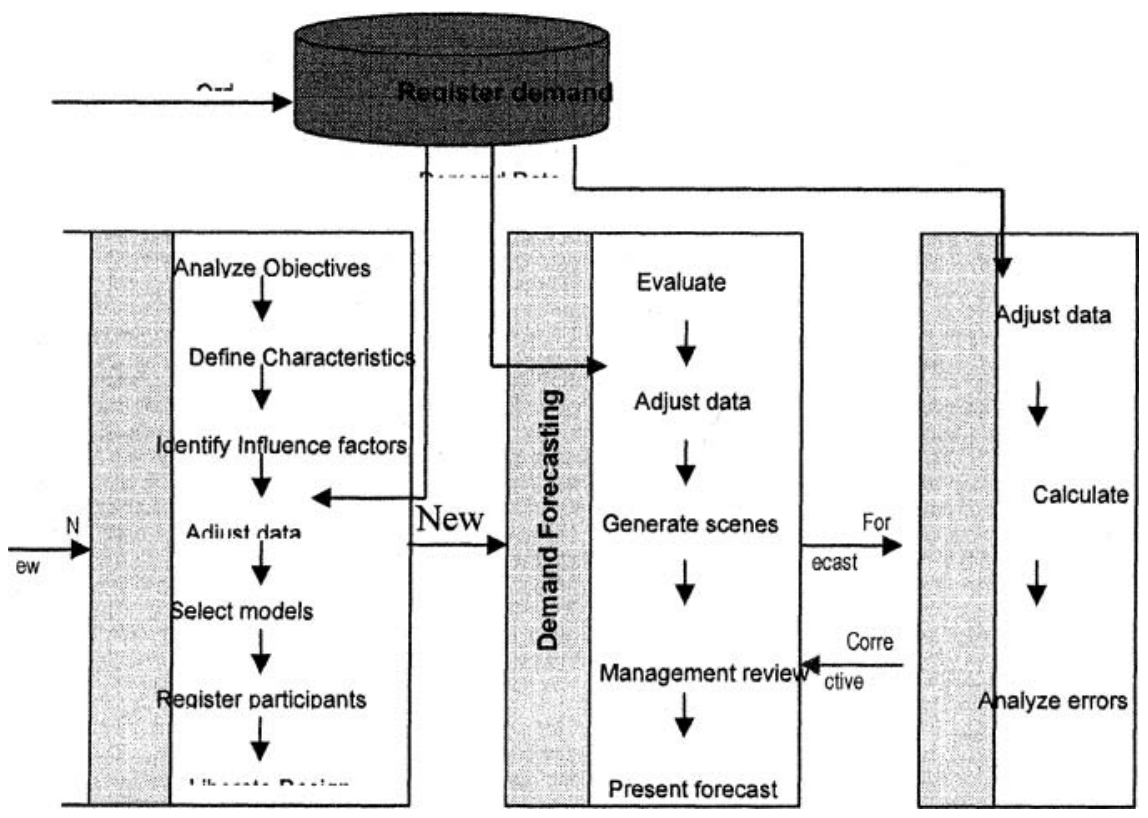

Fig. 1. Procedure considered by Santos [5]. 
The forecasting design possess seven activities such as: (1) define objectives (type, stated period, family); soon after (2) define forecasting characteristics (horizon of time, region, type of customer); after that (3) identify influence external and internal factors such as: price of purchase, promotion of the competitors, launching of new products, description of purchases, as well as the action of the government, action of competitors, climate, level of economic activity. Next it is adjusted (4) demand data in agreement with the defined factors; it follows (5) the election of models that will be used in it the calculation of the demand forecasting (mathematical and statistical models); later it is defined (6) participants of the forecasting, or either the sectors that participate of the forecasting process. In this point it is important to detach that the people who participates in the forecasting have the responsibility for the preparation of the demand forecasting, and generally it is the ones of the marketing or sales sectors, however the staff of the PCP also must participate because it is a sector directly affected by the forecasting. Moreover, in small-to-medium enterprises, generally speaking, there is no specialisation of the activities, so the staff of the PCP is also responsible for these attributions as well as the purchases and forecasting elaboration. Finally (7) to liberate the design for the accomplishment of the actual forecasting.

After liberating the forecasting process design to the participants, the system generates a solicitation of forecasting process to start it. This is composed of five activities. The data of the solicitation of the forecasting process are, initially, (1) analysed, where occasionally, it can be necessary (2) to adjust the most recent data of the demand. Later it must (3) be generated the scenarios, where the system will calculate the forecasting in accordance with the selected mathematical models, and the hypotheses considered in the model, as defined in the demand forecasting design described previously. After that it is accomplished the (4) management review, then if the manager judges necessary, there will be done an adjustment for the values of the scenario that will define the final forecasting and must be done a description, justifying the new adjustment and finally (5) to present the forecasting.

Results of the demand forecasting calculated from the products demand are evaluated in the procedure of accompanying the errors. This procedure possess three activities that are: (1) to calculate the error, where the system will calculate automatically at all final period the errors of forecasting, and in case that the error exceeds the acceptable limits, corrective actions must be taken in order to adjust the forecasting process or even though to review the design. Next, in case it is necessary, it is done a (2) new adjustment of data, and finally (3) it is analysed the errors leaving registered in the system the reasons of the error and control actions that must be taken in order to correct the forecasting.

For the development and documentation of the models are used support computing tools. In the modelling processes of the demand forecasting it was used CimTOOL software and in the modelling of the information system it was used Rational Rose software.

The information system to aid the demand forecasting implements the reference model of the demand forecasting process. It has the objective to supply a view of how this process can be carried through and adapted in accordance with the 
company, allowing the managers to analyse the demand, to adjust the production and moreover to serve as study and simulation of the products demand forecasting of the enterprise.

\section{Discussion}

Some business practices point to ERP (Enterprise Resources Planning) flexibility that the ERP systems allow the decisions and the data base from all parts of the organization be reflected on the planning and control systems of the rest of the organization [14]. This can be obtained through a vertical form with sets of preconfigured standard systems which allow adjusting to combine specific or partial requirements as it is proposed by CIMOSA [1].

Many studies have been developed to promote an open modelling architecture to general model; however, the data collection of a company linked to an ERP data base can present greater flexibility and be more efficient in model maintenance. Allied to intelligent methods of analysing it can provide answers to the problems that were not considered during the design. The model proposed by Dewhurst [9] can be analyzed by multiple perspectives, if applied OLAP tools (on-line analytic).

It is used to integrate the current necessities of the market and provide the access to the competitive advantages as connectiveness, collaboration among enterprises, BI (Business Intelligence) and low cost application. Considering the knowledge acquisition of the enterprise and its transformation into an integrated information system, it is extremely important the employment of techniques for the survey and elicitation of what is intended to design, although each technique highlights one or even several aspects of the problem at question and does not represent the success guarantee.

On the other hand, initiatives go forward to a challenge of reaching a solution which integrates better practices and extends the model to open architectures of low cost and more flexible or adaptable as the ERP5 project proposes. The aim of the ERP5 project is to draw and develop a complete configuration of ERP software components and provide sufficient information (juridical, social, theoretical) in order to able everyone to understand and implement the ERP in small-to-medium enterprises $[15,16]$.

ERP5 is an audacious design and seeks to associate the great necessity of the enterprises in obtaining a solution of low cost $[15,16]$ implements the ERP concepts, Internet (e-business) and abstract enterprise (multi-enterprise). However the existing gap between the knowledge acquisition and design performance per se still represents a great challenge. 


\section{Conclusions}

This study approached a proposal of approximating the operations and enterprise information systems detaching the usage of some pieces of information in the enterprise. To the enterprise reference models could be used to give safe, rapid and economical directions to the designs of particular models of enterprise systems.

The process modeling through CIMOSA provided an organized set of pieces of information, facilitating the mapping to design a system with UML. The prototype based on a reference model as proposed will be able to be customized, that is, adapted to answer to the particularities of a determined enterprise, and it will be used as an additional component of some managing enterprise system.

In the support system proposed to the model during the accomplishment of the activities of the forecasting processes are also present some fields where analysis and description will be done. The systematic register of these data allows placing the decisions and conclusions in accordance with the time (period) and the context. Allowing a comparison of the results of the mathematical models selected with the pieces of information of qualitative analysis, executed by experts it is possible to assess and test new parameters and/or models, aiming to approximate to the reality the results forecasted and make possible the decision with a greater consistency.

With the aim to have influence on and favour potentialities of future works it might be used solutions as Data warehouse to, for example, transform the operational data into strategic ones, transforming this into an automatic decision support system, and use extraction tools on information as data Mining [5].

\section{References}

1. C. Møller, ERP II: A Conceptual Framework for Next-Generation Enterprise Systems? Journal of Enterprise Information Management 18(4), 483-497 (2005).

2. D.M.R. Ferreira and J.J.P. Ferreira, Towards a Workflow-Based Integration Architecture for Business Networking, Business Process Management 11(5), pp. 517-531 (2005).

3. F.B. Vernadat, Enterprise Modeling and Integration, Principles and Applications (Chapman \& Hall, Londres, 1996).

4. K. Kosanke, F.B. Vernadat, and M. Zelm, CIMOSA: Enterprise Engineering and Integration. Computers in Industry 40(2), 83-97 (1999).

5. L.R Santos, Enterprise Processes and Information System Modeling: An Application to Product Demand Forecasting, M. Sc. Thesis, State University of North Fluminense, Brazil, 2001.

6. F.B. Vernadat, Enterprise Modeling: Objectives, Constructs \& Ontologies, Tutorial held at the EMOI-CAISE Workshop, Riga, Latvia, June 7, 2004.

7. H. Shen, B. Wall, M. Zaremba, Y. Chen, and J. Browne, Integration Of Business Modelling Methods for Enterprise Information System Analysis and User Requirements Gathering, Computers in Industry 54(3), 307-323 (2004).

8. R.S. Aguilar-Savén, Business Process Modelling: Review and Framework, International Journal of Production Economics 90(2), 129-149 (2004). 
9. F.W. Dewhurst, K.D. Barber, and M.C. Pritchard, In Search of a General Enterprise Model, Management Decision 40(5), 418-427 (2002).

10. G. Booch, J. Rumbaugh, and I. Jacobson, Unified Modeling Language User Guide (Addisson Wesley, Massachusetts, 1999).

11.C. Larman, Applying UML and Patterns (Prentice Hall PTR, New York, 2004).

12. CIMOSA Association, Enterprise Integration - Constructs for Enterprise Modeling EN/ISO 19440: 2003 - ISO TC184/SC5/WG1 Naples, Florida, 2003.

13. K. Nikolopoulos and V. Assimakopoulos, Theta Intelligent Forecasting Information System, Industrial Management \& Data Systems 103(9), 711-726 (2003).

14. A. Serrano and M. den Hengst, Modeling the Integration of BP and IT Using Business Processes Simulation, Journal of Enterprise Information Management, 18(6), 740-759 (2005). 15. J-P. Smets-Solanes and R.A. Carvalho, An Abstract Model for an Open Source ERP System: The ERP5 Proposal, Enegep, Brazil, 2005.

16. J-P. Smets-Solanes, ERP5: A Technical Introduction; http://ww.erp5.org 